Final Report for Period: $\quad$ 09/2000 - 08/2003

Principal Investigator: Mori, Warren B.

Organization: U of Cal Los Angeles

Title:

Continuation of Full-Seale Three-Dimensional Numerical Experiments on High-Intensity Particle and Laser Beam-Matter Interactions
Submitted on: 09/18/2003

Award ID: 0078508

\title{
Senior Personnel
}

\section{Project Participants}

Name: Mori, Warren

Worked for more than 160 Hours: Yes

Contribution to Project:

Name: decyk, viktor

Worked for more than 160 Hours: Yes

Contribution to Project:

manages parallel code development and works on appleseed cluster

Post-doc

Name: dodd, evan

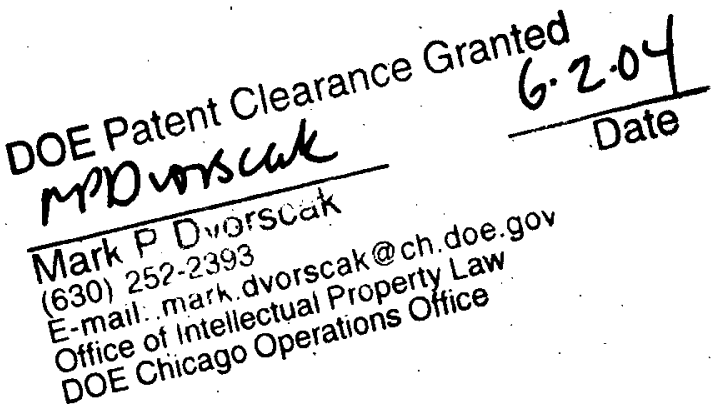

Worked for more than 160 Hours: Yes

Contribution to Project:

Used OSIRIS to cayy out full-scale simulations of PWFA experiments and studied electron hosing of GeV beams for realistic beam profiles.

Name: Ren, Chung

Worked for more than 160 Hours: Yes

Contribution to Project:

Studied 3D wakes from beam drivers and is continuing to

develope wakefield theory for magnetized plasmas. Beginning

to study the absorption of ultra-intense lasers on overdense

plasma slabs.

Name: Tsung, Frank

Worked for more than 160 Hours: Yes

Contribution to Project:

Maintains the code osiris and studies how short pulses evolve while propagating through underdense plasmas. Modeling self-modulated wakefield experiments in three-dimensions.

\section{Graduate Student}

Name: Wang, Shoquin

Worked for more than 160 Hours: No

Contribution to Project:

Studied spontaneous $x$-ray radiation from betatron motion when an intense electron beam self-consistently forms an ion channel.

Name: Tonge, John

Worked for more than 160 Hours: No

Contribution to Project:

Studied colliding electron-positron clouds.

Neme: Huang, Chuing-Kun

Worked for more than 160 Hours: No 


\section{DISCLAIMER}

This report was prepared as an account of work sponsored by an agency of the United States Government. Neither the United States Government nor any agency Thereof, nor any of their employees, makes any warranty, express or implied, or assumes any legal liability or responsibility for the accuracy, completeness, or usefulness of any information, apparatus, product, or process disclosed, or represents that its use would not infringe privately owned rights. Reference herein to any specific commercial product, process, or service by trade name, trademark, manufacturer, or otherwise does not necessarily constitute or imply its endorsement, recommendation, or favoring by the United States Government or any agency thereof. The views and opinions of authors expressed herein do not necessarily state or reflect those of the United States Government or any agency thereof. 


\section{DISCLAIMER}

Portions of this document may be illegible in electronic image products. Images are produced from the best available original document. 
Contribution to Project:

Developing a 3D parallel quasi-static PIC code called quickPIC.

Name: Lu, Wei

Worked for more than 160 Hours: Yes

Contribution to Project:

Using osiris to model plasma wakefield acceleration. The parameters are of relevance of a future experiment at SLAC.

Name: Fahlan, Jay

Worked for more than 160 Hours: No

Contribution to Project:

A beginning student who will model the interaction of an intense laser on a solid.

Name: Winjum, Benjamin

Worked for more than 160 Hours: Yes

Contribution to Projeet:

Used the code OSIRIS to study underdense laser-plasma interactions

Name: Zhou, MiaoMiao

Worked for more than 160 Hours: Yes

Contribution to Project:

Used the code quickPIC to study beam-plasma interactions

Name: Tzoufras, Michael

Worked for more than 160 Hours: Yes

Contribution to Project:

Used the code OSIRIS to study laser-plasma interactions

\section{Undergraduate Student}

Technician, Programmer

Other Participant

Research Experience for Undergraduates

\section{Organizational Partmers}

Other Collaborators or Contacts

Tom Katsouleas USC

L.O.Silva IST Portugal

J.C.Adan Ecole Polytechnique

Tom Antonsen, U.Maryland

\section{Activities and Mndings}

Resenrch and Education Activities:

Major research activities:

1.Continued development of the full 3D parallel PIC code

OSIRIS.

2.Parallel computing using OSIRIS

a. Modeled the PWFA experiment e-157.

b. Modeling LWFA experiments. 
c. Modeling SMLWFA experiments.

d. Modeling positron wakes.

e. Modeling electron-positron cloud interactions.

f. Modeling the electron bosing instability.

b. Modeling overdense laser plasma interactions.

3. Developing theoretical models for the mutual interaction of lasers in plasmas.

4. Developed a new 3D parallel quasj-static PIC code called quickPIC.

5. Developing tools for our appleseed cluster.

6. Developing theoretical models for Inser-plasma interactions in magnetized plasmas.

7. Started to use quickPIC to study

a. hosing of electron beams

b. positron wakes in uniform and hollow plasmas

Findings:

Major findings:।

Mutual attraction of lasers in plasmas: braided light:

We continued to study the mutual attraction of lasers in plasmas. We showed using a variational principle that there is a profound difference when the nonlinearity is not instantaneous. Furthermore, we found that the lasers can repel when the coherence between the two lasers is taken into account.

Refiraction of a particle beam:

We found that when a particle beam leaves a plasma gas interace it bends due an impulse from the collective fields at the plasma edge.

Electron beam hosing:

We have studied the electron hosing of GeV beams. We find that while bosing occurs it is with a lower growth nte than simple theory predicts. Furthermore, we find that when gross hosing occurs it does not pecessarily destroy the wake.

Development of quickPIC:

We have developed a new 3D parallelized quasi-static PIC code. Currently the code is tailored for studying the interaction of highly relativistic and narrow particle beam-plasma interactions. The code imbeds a 2D parallel PIC code inside a 3D parallel PIC code.

Positron wakes:

We studied plasma wakefield excitation by positron beams in a regime for which the plasmn dynamics are highly nonlinear. We used OSIRIS for this study. In the electron driver case plasma electrons are blown out while in the positron driver case they are pulled in. Although analogous, the two wakefield cases are quite different in terms of their amplitudes, wavelengths, wakeforms, transverse profiles, and plasm density dependence. In a homogenous plasma, nonlinear positron wakes are smaller than those of the corresponding electron case. However, hollow channels are shown to enhance the amplitude of the positron wakes.

Self-modulated laser wakefiled acceleration:

We studied the SMLFWA of 35 fs laser pulses. We modeled 
ongoing experiments in their full scale in 1,2, and 3 dimensions. We found that the the number of electrons and the maximum energy of an accelerated electron both decrease as the plasma density is raised. This was consistent with published experimental data. Furthermore, we find that the at the highest density (n/nc=.1) that the laser is completely absorbed within 300um.

Laser wakefield acceleration in cm long plasma channels: We performed full scale 3d simulations of LWFA in a channel. We found that short pulse, $50 \mathrm{fs}$, moderately intense laser, $-15 T W$, could be focused into a leaky channel of density $-5 \times 10^{\wedge} 18 \mathrm{~cm}-3$ and maintain its intensity for a cm. Furthermore, the laser's intensity evolves due to a combination of photon deceleration and group velocity dispersion so that it eventually leads to selfinjection of electrons from the channel walls. These electrons eventually reach $.84 \mathrm{GeV}$ energies.

Overdense laser plasma interactions:

We studied that generation of energetic protons when an intense short-pulse laser pulse hits a overdense plasma slab.

We found a new acceleration mechaniam. Initially electrons are accelerated across the slab. Some leave and others reflect from the back side and retum to the front side. At this time an ion acoustic shock is launched that traps and accelerated protons to relativistic energies. This work is being done in collaboration with the group in Portugal.

PWFA simulations:

We are modeling the experimenal parameters of a proposed experiment at SLAC called E-164. We find that for these parameters that the wake amplitude that the optimum plasma density is above that predicted from linear theory. Futhermore, we find that a small number of electrons are self-trapped and this number can be much larger due to secondary impact ionization.

\section{Afterbumers:}

We made preliminary studies of key aspects of the afterburner concept. We found that for these parameters that the hosing instability is greatly supressed. We also found a beamloading regime where a loaded transformer ration exceeding 2 is possible.

\section{Training and Development:}

Research on this project is training both post-docs and students in the use of massively parallel computing in high-energy density laser and beam plasma interactions.

\section{Outreach Aetivities:}

Our development of an easy to use appleseed cluster continues to have impact to $\mathrm{k} 12$ students. In fact, a sixth grader has downloaded our software to network two macintosh computers together. We are continually being contacted by high-schools and universities about how to build an appleseed cluster.

\section{Journal Publications}

S.Lee, T.Katsouleas, R.G.Hernker, E.S.Dodd, and W.B.Mori, "Plasma-wakefield acceleration of a positron beam", Phys Rev, E rapid communications, p. 045501, vol. 64, (2001). Published 
Chengkun Huang, Viktor Decyk, Shuogin Wang, Evan S.Dodd, Chuang Ren, Warren B. Mori, Tom Katsouleas, and Tom Antonsen Jr., "quickPIC: A parallelized quasi-static PIC code for modeling

plasma wakefield acceleration", Proceedings of the 2001 Particle accelerator conference, p. 4005, vol. 5, (2001). Published

E.S.Dodd, R.G.Hemker, C-K. Huang, C.Ren, W.B.Mori, S.Lee, and T.Katsouleas, "Hosing and sloshing of short-pulse GeV class wakefield drivers", Phyical Review Letters, p. 125001, vol. 88, (2002). Published

Patric Muggli, S.Lee, T.Katsouleas, R.Assman, F.J.Decker, M.Hogan, R.Iverson, P. Raimondi, R.Siemann, D.Walz, B.Blue, C.Clayton, E.Dodd, R.Fonseca, R.Hemker, C.Joshi, K.Marsh, W.Mori, S. Wang, "Collective refraction of a beam of electrons at a plasma-gas interface", Nature, p. 43, vol. 411, (2001). Published

F.S.Tsung, C.Ren, L.O.Silva, R.G.Hemker, W.B.Mori and T.Katsouleas, "Generation of.high-intensity single cycle pulses using photon deceleration", Proceedings of the National Academy of Sciences, p. 29, vol. 99, (2002). Published

C.Ren, B.J.Duda, and W.B.Mori, "Braiding of two laser beams due to plasma wakefields", Physical Review E, p. 067401, vol. 64, (2001). Published

Ren C, et al., "On the mutual interaction between laser beams in plasmas", Physics of Plasmas, p. 2354, vol. 9, (2002). Published

Clayton CE et al., "Transverse envelope dynamies of a $28.5-\mathrm{GeV}$ eeletron beam in

a long plasma", Physical Review Letters, p. 154801, vol. 88, (2002). Published

Wang Shuoquin et al., "X-ray emission from betatron motion in a plasma wiggler", Physical Review Letters, p. 135004, vol. 88, (2002). Published

Lee S. et al., "Energy doubler for a linear collider", Physical Review Special Topics-Accelerators and Beams, p. 01 1001, vol. 5, (2002). Published

Joshi C.et al., "High energy density plasma science with an ultrarelativistic

electron beam". Physics of Plasmias, p. 1845, vol. 9, (2002). Published

M. J. Hogan, C. E. Clayton, C. Huang, P. Muggli, S. Wang, B. E. Blue, D. Walz, K. A. Marsh, C. L. O'Connell, S. Lee, R. Iverson, F-J. Decker, P. Raimondi, W. B. Mori, T. C. Katsouleas, C. Joshi and R. H. Siemann, "Ultrarelativistic-Positron-Beam Transport through Meter-Scale Plasmas", Physical Review Letters, p. 205002-1, vol. 90, (2003). Published

B. E. Blue, C. E. Clayton, C. L. O'Connell, F-J. Decker, M.J. Hogan, C. Huang, R. Iverson, C. Joshi, T. C. Katsouleas, W. Lu, K. A. Marsh, W. B. Mori, P. Muggli, R. Siemiann, and D. Walz, "Plasma-Wakefield Acceleration of an Intense Positron Beam", Physical Review Letters, p. 214801-1, vol. 90, (2003). Published

C. O'Connell, F-J. Decker, M. J. Hogan, R. Iverson, P. Raimondi, R. H. Siemann, D. Walz, B. Blue, C. E. Clayton, C. Joshi, K. A. Marsh, W. B. Mori, S. Wang, T. Katsouleas, S. Lee, and P. Muggli, "Dynamic focusing of an electron beam through a long plasma", Phys. Rev. Special Topics-Accelerators and Beams, p. 121301-1, vol. 5, (2002). Published

\section{Books or Other One-time Publications}

\section{Web/Internet Site}

URL(s):

exodus.physics.ucls.edu

Description: 


\section{Other Specilic Produets}

\section{Contributions}

Contributions within Discipline:

Contributions to Other Disciplines:

Contributions to Human Resource Development:

Contributions to Resources for Research and Education:

Contributions Beyond Science and Engineering:

\section{Categories for which nothing is reported:}

Organizational Partners

Any Book

Any Product

Contributions: To Any within Discipline

Contributions: To Any Other Disciplines

Contributions: To Any Human Resource Development

Contributions: To Any Resources for Research and Education

Contributions: To Any Beyond Science and Engineering 